\section{RSP}

http://www.rsp.fsp.usp.br/
Revista de Saúde Pública

\title{
O processo de implantação das unidades de pronto atendimento no Brasil
}

\author{
Gisele O'Dwyer', Mariana Teixeira Konder", Luciano Pereira Reciputti"', Mônica Guimarães Macau \\ Lopes $^{\mathrm{IV}}$, Danielle Fernandes Agostinho"II, Gabriel Farias Alves ${ }^{\mathrm{V}}$ \\ ' Fundação Oswaldo Cruz. Escola Nacional de Saúde Pública. Departamento de Administração e Planejamento \\ em Saúde. Rio de Janeiro, RJ, Brasil \\ " Universidade Estadual do Rio de Janeiro. Faculdade de Ciências Médicas. Departamento de Clínica Médica. \\ Rio de Janeiro, RJ, Brasil \\ III Fundação Oswaldo Cruz. Iniciação Científica. Escola Nacional de Saúde Pública. Departamento de \\ Administração e Planejamento em Saúde. Rio de Janeiro, RJ, Brasil \\ iv Ministério da Saúde. Secretaria de Atenção à Saúde. Departamento de Ações Programáticas e Estratégicas. \\ Brasília, DF, Brasil \\ $\checkmark$ Fundação de Apoio à Pesquisa do Estado do Rio de Janeiro Iniciação Científica. Escola Nacional de Saúde \\ Pública. Departamento de Administração e Planejamento em Saúde. Rio de Janeiro, RJ, Brasil
}

Correspondência:

Gisele O'Dwyer

Departamento de Administração e Planejamento em Saúde - ENSP Av. Leopoldo Bulhões, 1480 Sala 727 Manguinhos

21041-210 Rio de Janeiro, RJ, Brasil E-mail: odwyer@ensp.fiocruz.br

Recebido: 14 jun 2016

Aprovado: 16 jan 2017

Como citar: O'Dwyer G, Konder MT, Reciputti LP, Lopes MGM, Agostinho DF, Alves GF. O processo de implantação das unidades de pronto atendimento no Brasil. Rev Saude Publica. 2017:51:125.

Copyright: Este é um artigo de acesso aberto distribuído sob os termos da Licença de Atribuição Creative Commons, que permite uso irrestrito, distribuição e reprodução em qualquer meio desde que o autor e a fonte originais sejam creditados.

\section{RESUMO}

OBJETIVO: Analisar o processo de implantação das unidades de pronto atendimento no Brasil. MÉTODOS: Realizou-se análise documental, entrevistas com 24 coordenadores estaduais de urgência e um painel de especialistas. Analisaram-se questões relativas a: antecedentes e trajetória da política; atores envolvidos na implantação; processo de expansão; avanços, limites e dificuldades de implantação; e capacidade de coordenação estadual. Utilizou-se o referencial teórico da análise da conduta estratégica da teoria da estruturação de Giddens.

RESULTADOS: As unidades de pronto atendimento foram implantadas a partir de 2007, inicialmente na região Sudeste, e em 2016 existiam 446 unidades de pronto atendimento considerando todas as regiões. Atualmente, há 620 unidades de pronto atendimento em construção, indicando expectativa de expansão. $\mathrm{O}$ financiamento federal foi um forte indutor da implantação. Os estados planejaram suas unidades de pronto atendimento, mas a existência de negociação direta entre os municípios e a União contribuiu com o significativo número de unidades de pronto atendimento construídas que não funcionam. Em relação à rede de urgência, há tensão com o hospital pela insuficiência de leitos no país, gerando internação na unidade de pronto atendimento. A gestão das unidades de pronto atendimento é predominantemente municipal, com a maioria das unidades de pronto atendimento localizadas fora das capitais e classificadas como Porte III. Os principais desafios identificados foram: o sub-financiamento e a dificuldade de contratar médicos.

CONCLUSÕES: A unidade de pronto atendimento tem o mérito de ter recursos tecnológicos e ser arquitetonicamente diferenciada, mas só será bem-sucedida dentro de uma rede de urgência. A indução federal gerou respostas contraditórias, pois nem todos os estados consideram a unidade de pronto atendimento como prioritária. O fortalecimento da gestão estadual foi identificado como desafio para a implantação da rede de urgências.

DESCRITORES: Serviços Médicos de Emergência, recursos humanos. Gestor de Saúde. Administração de Serviços de Saúde. Gestão de Recursos. Gestão em Saúde. Política de Saúde. 


\section{INTRODUÇÃO}

No início dos anos 2000, estabeleceu-se no âmbito do Ministério da Saúde a organização de uma política nacional de atenção às urgências, com a implantação de novos componentes, como os serviços de atendimento móvel de urgência (SAMU) e as unidades de pronto atendimento (UPA). Iniciar a implantação pelo pré-hospitalar foi uma decisão acertada, já que as experiências nacionai ${ }^{9,12,15}$ e internacionais ${ }^{2,21}$ mostram o impacto positivo desse atendimento. Cronologicamente, a implantação dos componentes pré-hospitalares ocorreu de forma desarticulada ${ }^{15}$, em três momentos distintos ${ }^{10}$ : até 2002 - regulamentação inicial; 20032008 - ênfase no SAMU; a partir de 2009 - ênfase na UPA. Em 2011, definiu-se a necessidade de uma rede de urgência, com regionalização e reorganização dos serviços pré-existentes ${ }^{13}$.

As UPA, principal componente fixo de urgência pré-hospitalar, são unidades intermediárias entre a atenção primária e as emergências hospitalares. Classificam-se em três diferentes portes, de acordo com a população referenciada, a área física, o número de leitos disponíveis, a gestão de pessoas e a capacidade de atender ${ }^{14}$.

As portarias editadas ao longo dos anos previram UPA estrategicamente integrada em redes de atenção às urgências ${ }^{6}$. A necessária coexistência com um SAMU e a obrigatoriedade de expandir a cobertura de atenção primária através da Estratégia Saúde da Família (ESF) são condicionalidades que procuram fortalecer a visão de rede e impelir os gestores a investir em outros componentes da rede de urgências. Assim demarca-se a pretendida resolutividade da UPA, diferenciando-se dos antigos prontos socorros, produtores de "consultas de emergência" pouco resolutivas.

Pesquisa nacional ${ }^{3}$ identificou que todas as UPA fazem classificação de risco e que são estruturalmente bem equipadas. Os principais desafios encontrados englobavam a gestão profissional e a "internação" dos pacientes ${ }^{3}$. O Rio de Janeiro, primeiro estado a implantar UPA antes das portarias federais e criador desse nome, constituiu seu parque de unidades contando com uma convergência de interesses que permitiu sua rápida expansão ${ }^{9}$. Esse estado expandiu as UPA de forma acelerada sem efetiva organização da rede de urgência? Outra pesquisa evidenciou os limites na gestão do trabalho em saúde, expressas nas formas de contratação, fixação e capacitação dos profissionais nas UPA ${ }^{11}$ com implicações na qualidade do atendimento. Apesar das dificuldades apresentadas nos estudos ${ }^{7,911}$, as UPA representaram uma ampliação do acesso a consultas médicas e exames complementares, principalmente, em casos de menor complexidade?

Esta pesquisa teve por objetivo analisar o processo de implantação das UPA no Brasil, identificando: as regras e recursos que facilitaram sua implantação; os elementos dificultadores e facilitadores nesse processo; a influência dos agentes das diferentes esferas governamentais na implantação desses serviços; e a proposta de expansão das UPA.

\section{MÉTODOS}

Realizou-se análise da legislação, documentos e sites oficiais dos municípios, estados e federação, além de entrevistas com atores-chave e um painel de especialistas.

Foram entrevistados os coordenadores estaduais de urgência, que participaram de um painel de especialistas. Esses atores foram escolhidos por sua relevância para a assistência integral às urgências, para a negociação de pactos e metas e para a cooperação entre entes federativos. No total, 24 coordenadores estaduais do país aceitaram participar. As entrevistas basearam-se em um roteiro semiestruturado e ocorreram em 2013 e 2014. O painel de especialistas tratou de forma coletiva dos mesmos temas das entrevistas, oportunizando o compartilhamento de experiências. O painel ocorreu em dois dias (16 horas) no final de 2013 e contou com o coordenador geral de urgência e emergência do Ministério da Saúde e três integrantes da Rede Brasileira de Cooperação em Emergência 
(RBCE), instituição fundamental na formulação e implantação da política de urgência, além dos entrevistados. O material do painel e das entrevistas foi analisado conjuntamente, considerando-se: antecedentes e trajetória da política; atores envolvidos na implantação; processo de expansão; avanços, limites e dificuldades de implantação; e capacidade de coordenação estadual. Os documentos compuseram as regras e recursos disponíveis para a ação dos atores envolvidos. O plano de análise visou a identificar as circunstâncias estruturais disponíveis para a ação dos gestores e as estratégias que diferenciaram os estados, caracterizando a implantação das UPA no país.

Utilizou-se o referencial teórico da análise da conduta estratégica da Teoria da Estruturação de Giddens ${ }^{5}$, segundo o qual as práticas sociais podem ser entendidas como procedimentos, métodos ou técnicas hábeis executadas pelos agentes sociais, fazendo uso de regras e recursos estruturais ${ }^{5}$. As dimensões estruturais (a base de poder) são facilitadoras ou restritivas e os agentes as acessam para manipular e influenciar as interações sociais. São três as modalidades da estruturação, correlacionando as capacidades cognitivas dos agentes e as características estruturais: significação, que a partir de um esquema interpretativo é possibilitada pela comunicação; dominação, que depende da mobilização de recursos alocativos e autoritários; e legitimação, exercida a partir da sanção de normas.

Esse projeto foi aprovado pelo CAAE 0209.0.031.000-11.

\section{RESULTADOS E DISCUSSÃO}

As portarias referentes à formulação da Política de Urgência foram analisadas. Foi disponibilizado pelo Ministério da Saúde relatório que informava a esfera responsável pela gestão das UPA e consultada a Sala de Apoio à Gestão Estratégica da página do Ministério, principais fontes dos dados quantitativos descritivos.

\section{Antecedentes da Atenção Pré-Hospitalar Fixa}

Há décadas, o atendimento pré-hospitalar fixo foi assumido principalmente pelos gestores municipais que cumpriam esse papel assistencial de forma diversa. Grandes centros urbanos foram os precursores, contando com unidades abertas à demanda espontânea, mas dispondo predominantemente de estrutura e equipamentos para atendimento de baixa complexidade.

Destacam-se os serviços de pronto atendimento (SPA), as unidades de atendimento imediato (UAI), os prontos atendimentos médicos (PAM), os centros municipais de urgência e emergências (CMUM), os centros regionais de saúde (CRS). As unidades de assistência médica ambulatorial (AMA) do município de São Paulo representaram uma das alternativas anteriores à UPA. As AMA visavam a ampliar o acesso da população com quadros agudos de baixa e média complexidade, com compromisso de integrá-los à atenção primária ${ }^{19}$. Entretanto, identificou-se predomínio de unidades com integração insatisfatória ${ }^{19}$.

Antes das UPA, havia uma rede para o pré-hospitalar fixo não regulada pelo Estado que cumpria seu papel sem avaliação sistemática. Na maioria das vezes, essas unidades não eram expressivas em termos estruturais, não classificavam risco e produziam consultas pouco resolutivas.

Apesar de não serem unidades pré-hospitalares, os hospitais de pequeno porte eventualmente desempenharam esse papel, principalmente em municípios pequenos e do interior. Incapazes de cumprir sua função de unidade de internação, por deficiências estruturais e de recursos humanos, em algumas localidades, os hospitais de pequeno porte apenas proviam atenção de demanda espontânea, confirmando a sua ociosidade ${ }^{20}$.

O recurso federal e a regra para definição estrutural foram indutores para a UPA. Entretanto, os agentes municipais e estaduais já haviam definido suas estratégias para configuração da urgência pré-hospitalar fixa. 


\section{Implantação e Expansão das UPA}

A implantação das UPA se iniciou com uma portaria em 2008. O estado do Rio de Janeiro foi o pioneiro nesse processo, implantando a primeira UPA no país em 2007, antes da regulação federal. Nesse estado, a UPA foi priorizada na agenda governamental por uma confluência de fatores histórico-estruturais, político-institucionais e conjunturais, que possibilitou a implantação antes dos incentivos federais? .

Segundo definições normativas para implantação da UPA, havia a exigência da presença de um SAMU, de uma rede hospitalar de referência e de atenção primária com cobertura de $50 \%$ ou em desenvolvimento, em municípios com pelo menos 50 mil habitantes.

A maioria das UPA concentram-se em municípios com mais de um milhão de habitantes, com privilégio das regiões com melhores condições socioeconômicas e melhor oferta de serviços, como a região Sudeste ${ }^{3}$.

A Tabela 1 apresenta a expansão da UPA no país no período entre 2011 e 2016. Optou-se por esse período por caracterizar o início da indução federal pelo Programa de Aceleração do Crescimento (PAC). Até 2011, as UPA se concentravam na região Sudeste. Já em 2016, existe um número expressivo de UPA em todo o país. Das 485 UPA em 2011 (soma das construídas e em construção), 446 estão prontas e em funcionamento desde 2016. Em 2016 ainda havia expectativa de expansão - 620 UPA estavam em construção -, apesar das 132 construídas sem funcionar. São Paulo tem o maior parque de UPA em funcionamento, em construção e sem funcionar. Proporcionalmente, as regiões Norte e Centro-Oeste têm o maior número de UPA sem funcionar, considerando o total em funcionamento. O Norte foi conservador na implantação e expansão de UPA, com exceção do Pará.

Segundo os entrevistados, alguns fatores explicam esse cenário de expansão variada no Brasil: divergência entre interesses políticos e critérios técnicos; dificuldades financeiras ou operacionais na esfera municipal, como contratar médicos; insuficiência de recursos de custeio de origem federal; dificuldade de regionalização das UPA municipais; projetos de UPA que se mostraram equivocados posteriormente, por falta de planejamento; e falta de credibilidade da UPA e de apoio estadual.

Houve unanimidade sobre a insuficiência dos recursos federais para implantação e custeio, este último teoricamente compartilhado entre estado e município.

Os PAC 1 e PAC 2 foram importantes fontes de financiamento para a expansão das UPA. No PAC 2, alguns municípios foram selecionados independentemente dos planos regionais de urgência. Segundo os coordenadores estaduais, a tentativa de regionalizar a urgência ficou comprometida quando o estado foi alijado do processo de expansão, conforme fala a seguir: "Acho que foi uma ingerência que produziu algumas expectativas... o município diz eu quero a minha própria UPA... fragilizou um pouco a parte técnica... a política que financiou o PAC 2 definiu a expansão" (E14).

Outros estados apropriaram-se melhor da função de coordenador das demandas municipais: "Eu recebi vários ofícios da câmara dos deputados pedindo UPA para o município... sempre dava a mesma resposta... o critério é técnico, a necessidade da região" (E9).

A indução federal também produziu a transformação de serviços anteriormente financiados por estados ou municípios em UPA. "Os SPA (serviços de pronto atendimento) são 100\% custeados pelo estado. Eles vão receber recurso federal quando habilitarem como UPA”(E3).

Houve coordenador desfavorável à implantação do componente UPA na rede de urgência, o que não impediu sua implantação. Outros reconheceram a adequação e finalidade da UPA. "Eu acho a UPA fundamental para a rede" (E22).

O estado de São Paulo ilustra uma peculiaridade, já que seu parque de UPA está alocado fora da capital, evidenciando a independência do nível municipal. 
Os gestores estaduais foram enfáticos ao afirmar que a expansão do número de UPA, mais do que a implantação inicial, era uma pretensão federal. "Penso que estamos num número muito bom. Expandir não, mas colocá-las para funcionar é o maior desafio com a articulação com o sistema municipal onde estão inseridas" (E16).

As UPA, ao contrário do SAMU ${ }^{15}$, não respondiam a uma lacuna assistencial substantiva para a rede de urgências. Sua implantação dependeu mais de incentivo financeiro federal que, por ser caro aos municípios, produziu capacidades de respostas algumas vezes frustradas, na concepção dos gestores.

Tabela 1. Número de unidades de pronto atendimento (UPA) existentes, em construção e construídas sem funcionar por unidade federativa. Brasil, 2011 e 2016.

\begin{tabular}{|c|c|c|c|c|c|}
\hline \multirow[b]{2}{*}{ Unidades federativas } & \multicolumn{2}{|c|}{ Ano 2011} & \multicolumn{3}{|c|}{ Ano 2016} \\
\hline & UPA existente & $\begin{array}{l}\text { UPA em } \\
\text { construção }\end{array}$ & $\begin{array}{c}\text { UPA } \\
\text { existente }\end{array}$ & $\begin{array}{l}\text { UPA em } \\
\text { construção }\end{array}$ & $\begin{array}{c}\text { UPA } \\
\text { construídas sem funcionar }\end{array}$ \\
\hline \multicolumn{6}{|l|}{ Região Norte } \\
\hline Acre & 2 & 1 & 2 & 5 & 0 \\
\hline Amapá & 0 & 3 & 1 & 3 & 0 \\
\hline Amazonas & 0 & 9 & 1 & 4 & 0 \\
\hline Pará* & 1 & 20 & 11 & 40 & 13 \\
\hline Rondônia & 0 & 3 & 2 & 7 & 2 \\
\hline Roraima & 0 & 1 & 0 & 1 & 0 \\
\hline Tocantins* & 2 & 4 & 6 & 7 & 4 \\
\hline Total & 5 & 41 & 23 & 67 & 19 \\
\hline \multicolumn{6}{|l|}{ Região Nordeste } \\
\hline Alagoas & 0 & 6 & 8 & 8 & 2 \\
\hline Bahia & 1 & 32 & 29 & 52 & 12 \\
\hline Ceará & 0 & 19 & 26 & 26 & 9 \\
\hline Maranhão & 1 & 7 & 11 & 17 & 3 \\
\hline Paraíba & 1 & 6 & 10 & 18 & 2 \\
\hline Pernambuco & 14 & 6 & 18 & 28 & 7 \\
\hline Piauí* & 0 & 3 & 2 & 12 & 3 \\
\hline Rio Grande do Norte & 3 & 6 & 6 & 11 & 2 \\
\hline Sergipe & 0 & 0 & 4 & 7 & 0 \\
\hline Total & 20 & 85 & 114 & 179 & 40 \\
\hline \multicolumn{6}{|l|}{ Região Centro-Oeste } \\
\hline Distrito Federal & 1 & 8 & 6 & 4 & 0 \\
\hline Goiás* & 1 & 17 & 14 & 34 & 12 \\
\hline Mato Grosso* & 0 & 8 & 4 & 23 & 2 \\
\hline Mato Grosso do Sul & 0 & 4 & 7 & 7 & 2 \\
\hline Total & 2 & 37 & 31 & 68 & 16 \\
\hline \multicolumn{6}{|l|}{ Região Sudeste } \\
\hline Espírito Santo & 1 & 5 & 4 & 8 & 0 \\
\hline Minas Gerais & 8 & 43 & 45 & 47 & 10 \\
\hline Rio de Janeiro & 43 & 16 & 70 & 12 & 2 \\
\hline São Paulo & 13 & 95 & 99 & 145 & 19 \\
\hline Total & 65 & 159 & 218 & 212 & 31 \\
\hline \multicolumn{6}{|l|}{ Região Sul } \\
\hline Paraná & 9 & 22 & 34 & 30 & 6 \\
\hline Santa Catarina* & 2 & 12 & 10 & 21 & 6 \\
\hline Rio Grande do Sul* & 0 & 26 & 16 & 43 & 16 \\
\hline Total & 11 & 60 & 60 & 94 & 28 \\
\hline Total Brasil & 101 & 381 & 446 & 620 & 134 \\
\hline
\end{tabular}

Fonte: Departamento de Atenção Hospitalar e Urgência (DAHU)/Ministério da Saúde e Sala de Apoio a Gestão Estratégica (SAGE) 2011 e 2016.

* Estados com um número expressivo de UPA sem funcionar, provavelmente por algumas recém-construídas e ainda estarem sem condições de funcionamento por falta de profissionais e insumos. 
O gestor municipal apoiou-se no ente federal para a expansão das UPA e a maioria dos gestores estaduais sentiu-se impotente na execução dos seus planos estaduais de urgência.

\section{As Condições Estruturais para o Atendimento}

A principal característica da UPA é a adequação da sua estrutura, o conforto das instalações e a adequação dos equipamentos. Pela primeira vez, um componente do SUS foi proposto com grande exigência nos critérios estruturais. Esse foi um diferencial expressivo, segundo os entrevistados, em relação ao parque de prontos-socorros já existente. "Evoluiu do modelo de PS que não tinha resolutividade, espaço físico, equipe qualificada, material, medicamento" (E7).

Houve referência à suficiência dos recursos diagnósticos laboratoriais e acesso aos recursos de imagem, como observado em pesquisa nacional ${ }^{3}$. Entretanto, a manutenção das UPA eventualmente foi um problema. "Falta de manutenção dos equipamentos acaba precarizando a estrutura física" (E16).

Os sistemas de informação foram citados como um problema estrutural. "UPA não são informatizadas. Fazem relatórios manuais de tipo de atendimento" (E13).

Entretanto, a questão estrutural mais impactante foi a falta de profissionais. "Gargalo é recursos humanos" (E16).

Esse problema havia sido identificado nacionalmente ${ }^{3}$. A baixa qualificação dos profissionais foi exposta pelo fato de $34 \%$ dos médicos não possuir qualquer qualificação para atuação nas UPA. Além disso, o dimensionamento do número de profissionais estava aquém da demanda ${ }^{3}$. Outro estudo identificou: problemas de seleção e fixação; predominância de profissionais jovens e inexperientes; alta rotatividade de médicos; e UPA vista como trabalho temporário ${ }^{11}$.

Os problemas indicados por esses estudos foram reconhecidos pelos entrevistados, que ainda destacaram a incipiente qualificação e questões com o tipo de vínculo dos profissionais. "Os médicos vêm da ESF sem experiência. Dificuldade de capacitar" (E4). "Tem concursado do município, cedido do estado, tem contratado, é misto" (E7).

O ente federal foi protagonista de uma proposta de unidade estruturalmente diferenciada. Entretanto, nenhum dos três níveis governamentais foi capaz de equacionar positivamente a gestão de pessoas.

\section{A UPA e outros Componentes da Rede de Urgência}

O Quadro 1 apresenta e analisa como as UPA atuavam na integração com a atenção primária e o hospital. Destacam-se as seguintes funções para as UPA: complementar a atenção primária; complementar a emergência hospitalar; e funcionar como unidade de internação.

O componente pré-hospitalar fixo foi regulamentado como parte integrante da atenção primária. Como sua implantação ocorreu principalmente para desafogar os hospitais, muitos gestores entenderam a UPA como complementar ao hospital, como já evidenciado?

A maior parte dos atendimentos foram classificados como azuis ou verdes, ou seja, de baixa gravidade, como já identificado em estudos locais ${ }^{7,916}$ e nacionais ${ }^{3}$. Não há contradição no predomínio de urgências menos graves em unidades pré-hospitalares. Entretanto, assim como já indicado ${ }^{7,16}$, os entrevistados referiram que a expectativa dos profissionais é de atender prioritariamente pacientes graves. A preponderância de pacientes menos graves foi entendida como deficiência da atenção primária.

Considerando que a cobertura da ESF no país atingiu 61\% em 2015, os entrevistados avaliaram que as UPA não se relacionavam de forma efetiva com a atenção primária.

Alguns afirmaram que se a atenção primária estivesse bem estruturada, a UPA teria menos função. "Dispensável se há boa cobertura de atenção primária” (E9). 
Quadro 1. Análise da integração das UPA com outros componentes da rede.

\begin{tabular}{|c|c|c|}
\hline Situação identificada nas UPA & Avaliação dos entrevistados & Nossa análise \\
\hline $\begin{array}{l}\text { Atender urgências de baixa } \\
\text { e média gravidade de forma } \\
\text { complementar à atenção primária } \\
\\
\text { "UPA atende ambulatório na } \\
\text { maioria dos casos" (E5) } \\
\text { "Pacientes de UPA deveriam estar } \\
\text { na AB. Estado devia decidir investir } \\
\text { na AB" (E22) }\end{array}$ & $\begin{array}{l}\text { A insuficiência quantitativa } \\
\text { e qualitativa da atenção } \\
\text { primária produz esse } \\
\text { padrão de atendimento. }\end{array}$ & $\begin{array}{c}\text { A UPA tem sua função } \\
\text { no atendimento de baixa } \\
\text { gravidade por funcionar } 24 \mathrm{~h}, \\
\text { complementando a atenção } \\
\text { primaria. Entretanto, não deve ser } \\
\text { implantada de forma substitutiva à } \\
\text { atenção primária. }\end{array}$ \\
\hline $\begin{array}{l}\text { "UPA perto dos hospitais para } \\
\text { substituir o PS do hospital" (E8) }\end{array}$ & $\begin{array}{l}\text { As UPA foram implantadas para } \\
\text { substituir os pronto-socorros } \\
\text { dos hospitais e, nos municípios } \\
\text { menores, para substituir } \\
\text { hospitais ineficientes. }\end{array}$ & $\begin{array}{l}\text { O atendimento à demanda de } \\
\text { baixo risco em unidades pré- } \\
\text { hospitalares pode beneficiar } \\
\text { a emergência hospitalar que } \\
\text { deve priorizar os casos mais } \\
\text { complexos. Mas não é essa a } \\
\text { finalidade da UPA. }\end{array}$ \\
\hline $\begin{array}{l}\text { Funcionar como unidade de } \\
\text { internação }\end{array}$ & \multirow[b]{2}{*}{$\begin{array}{c}\text { Os entrevistados são contrários ao } \\
\text { "internamento" na UPA. Entretanto, } \\
\text { admitem que alguns gestores } \\
\text { contabilizam os leitos da sala } \\
\text { vermelha como leitos hospitalares, } \\
\text { inclusive de CTI. }\end{array}$} & \multirow[b]{2}{*}{$\begin{array}{c}\text { A única função da UPA no } \\
\text { atendimento ao paciente grave } \\
\text { é sua estabilização. A UPA } \\
\text { é } 24 \mathrm{~h} \text { por ser porta aberta } \\
\text { ininterrupta e não por tolerar } \\
\text { permanência do paciente por } 24 \mathrm{~h} \\
\text { aguardando leitos. }\end{array}$} \\
\hline $\begin{array}{l}\text { "UPA não deve funcionar como } \\
\text { hospital de baixa resolutividade } \\
\text { por falta de leitos" (E9) } \\
\text { "Paciente internado na UPA e UPA } \\
\text { não recebe AIH" (E13) } \\
\text { "UPA faz bem: atender a baixa } \\
\text { complexidade, referenciar a média, } \\
\text { estabilizar o quadro grave, mas } \\
\text { internar é ruim" (E14) }\end{array}$ & & \\
\hline
\end{tabular}

UPA: unidade de pronto atendimento; AB: atenção básica; PS: pronto socorro; AlH: Autorização de Internação Hospitalar; CTI: Centro de Tratamento Intensivo

Apesar do impacto que a atenção primária insuficiente produz na UPA, mais grave é o impacto da insuficiência hospitalar, o que tem gerado algumas distorções. "Alguns gestores acham que a UPA resolve a falta de hospitais" (E10).

Identificam-se dois graves problemas no parque hospitalar do país: uma concentração de leitos em hospitais de pequeno porte e uma tendência a diminuir o já subdimensionado número de leitos. Os leitos SUS sofreram diminuição (10,5\%) no período de 2005 a $2014^{4}$. Em 2014, o SUS contava com 1,56 leitos por 1.000 habitantes, bem menos que o parâmetro sugerido pelo Ministério da Saúde (MS) de 2,5 a 3/1.000, ainda bastante conservador". "Em vez de abrir portas, a política deveria qualificá-las e aumentar leitos" (E16).

Apesar da obrigatoriedade da referência hospitalar, a dificuldade de internar pacientes foi considerada o maior desafio para as UPA.

A permanência de pacientes mais de 24 horas nas UPA por falta de vaga impacta a qualidade do atendimento ${ }^{3}$. Mais de quatro horas para transferência do paciente para o leito hospitalar agrava seu quadro ${ }^{8}$. Essa longa permanência é uma questão relevante, que reflete as dificuldades da rede hospitalar e foi destacado no Quadro 1, ao indicar-se o risco de internação de pacientes na UPA.

Além do déficit absoluto de leitos, grande parte desses leitos localizam-se em pequenos hospitais, com baixa capacidade resolutiva e baixas taxas de ocupação. Entretanto, esses leitos são contabilizados, apesar da insuficiência técnica para sua utilização 4 . O problema não se restringe aos hospitais menores. Atinge também as principais referências para as UPA, hospitais com mais de 100 leitos. Os gestores conheciam bem essa realidade. "Problemas com tempo de permanência nos grandes hospitais. Precisa capacitar chefe de plantão, coordenadores de clínica, quebrar feudos" (E11).

A baixa oferta de leitos de referência para as UPA gerou distorções. Alguns gestores tratavam os leitos de maior complexidade da sala vermelha como leitos hospitalares. 
Foram identificadas estratégias de enfrentamento do problema. Desde repasse financeiro diferenciado até o fortalecimento na gestão de hospitais de pequeno porte e filantrópicos. "Houve contrato com leitos de rede filantrópica. Recursos para leitos novos e leitos qualificados” (E11).

No nível federal, as iniciativas para o enfrentamento do problema hospitalar ainda são frustradas. A consequência é a superlotação nas emergências hospitalares dos grandes hospitais de referência. Essa superlotação gera pressão da porta de entrada ${ }^{1}$ e a UPA pode ser essencial por ser outra opção de acesso aberto. Outro componente da superlotação ${ }^{1}$, a capacidade de o hospital absorver o paciente da emergência, liberando leitos, impacta a UPA, demandante desse leito.

As dificuldades estruturais da atenção primária e hospitalar não favoreceram a configuração de uma rede de urgência onde a UPA estivesse integrada.

\section{A Gestão da UPA e a Interação entre os Entes Federados}

A Quadro 2 ilustra como atores municipais e estaduais mobilizaram recursos, legitimaram e significaram as UPA como componentes da rede de urgência, construindo novos contextos ${ }^{5}$, onde UPA podem ser implantadas a partir de planos estaduais em acordo com o município ou por decisão municipal autônoma. Evidenciou-se que a significação da UPA enquanto modalidade de estruturação não correspondeu à expectativa de um componente integrado à rede de urgência, mas significou uma nova porta de acesso no sistema. Foi legitimada quando foi identificada como componente fundamental, capaz de cumprir uma de suas funções que é ser complementar à atenção primária. A modalidade de estruturação dominação evidenciou que a mobilização das capacidades materiais e autoritativas gerou um padrão de implantação que algumas vezes foi mais político do que técnico, com pouca governança estadual.

Quadro 2. Análise das modalidades de estruturação e da ação dos entes federativos segundo cenário encontrado e falas dos gerentes.

\begin{tabular}{|c|c|c|c|}
\hline Categoria da teoria da estr & uturação & Cenário encontrado & Falas dos gestores \\
\hline \multirow{4}{*}{$\begin{array}{l}\text { Modalidades de } \\
\text { estruturação }\end{array}$} & $\begin{array}{l}\text { Significação - reflete um } \\
\text { esquema interpretativo }\end{array}$ & $\begin{array}{l}\text { UPA como componente que possibilita acesso } \\
\text { versus } \\
\text { UPA como componente } \\
\text { isolado na rede }\end{array}$ & $\begin{array}{l}\text { "Deve dar acesso, atender a baixa } \\
\text { complexidade, referenciar a média, estabilizar o } \\
\text { quadro grave" (E10 e E14) } \\
\text { "Retrocesso por ser isolada. } \\
\text { Quebra o modelo da AB" (E9) }\end{array}$ \\
\hline & & & "Eu acho a UPA fundamental para a rede" (E22) \\
\hline & $\begin{array}{l}\text { Legitimação - } \\
\text { cumprimento das normas }\end{array}$ & $\begin{array}{l}\text { UPA como componente integrado na rede, de } \\
\text { acordo com as portarias }\end{array}$ & $\begin{array}{l}\text { "Profissionais entendem a importância de } \\
\text { atender a urgência de baixa complexidade de } \\
\text { forma complementar à } A B^{\prime \prime} \text { (E15) }\end{array}$ \\
\hline & $\begin{array}{l}\text { Dominação - mobilização } \\
\text { de recuros alocativos } \\
\text { (capacidades materiais) e } \\
\text { autoritativos (relação entre } \\
\text { pessoas) }\end{array}$ & $\begin{array}{c}\text { Implantação de unidades necessárias } \\
\text { versus } \\
\text { Implantação política }\end{array}$ & $\begin{array}{l}\text { "Existe articulação política. O governo } \\
\text { do estado está solicitando as UPA } \\
\text { junto com o município" (E11) } \\
\text { "Meta política da Casa Civil para 2013. Manda o } \\
\text { MS executar sem o estado" (E12) }\end{array}$ \\
\hline \multirow[t]{2}{*}{$\begin{array}{l}\text { Agencialismo - } \\
\text { conhecimento e ação dos } \\
\text { atores institucionais }\end{array}$} & Ação do ator municipal & $\begin{array}{l}\text { Gestores municipais utilizando recursos } \\
\text { alocativos e autoritativos }\end{array}$ & $\begin{array}{c}\text { "O gestor municipal não tem onde lavar } \\
\text { roupa, fazer comida e resolve colocar tudo } \\
\text { isso na UPA, arcando com o financiamento. } \\
\text { Muda o gestor que não entende porque a } \\
\text { UPA é tão grande e onerosa, um fardo para a } \\
\text { estrutura do município" (E16) } \\
\\
\text { "Médicos saíram com a mudança do gestor. } \\
\text { Tem trombolítico e os novos médicos } \\
\text { não sabem usar" (E5) }\end{array}$ \\
\hline & Ação do ator estadual & $\begin{array}{l}\text { Gestores estaduais realizam o plano de } \\
\text { urgência e definem suas prioridades de gestão e } \\
\text { necessidades para o estado }\end{array}$ & $\begin{array}{l}\text { "Plano estadual para abrir leitos de UTI em cada } \\
\text { regional de saúde. Contrapartida do tesouro" (E9) }\end{array}$ \\
\hline
\end{tabular}

UPA: Unidade de Pronto Atendimento; AB: Atenção Básica; MS: Ministério da Saúde; UTI: Unidade de Terapia Intensiva 
No que diz respeito ao ente gestor das UPA, as regiões têm a maioria de suas UPA sob gestão municipal, com exceção do Nordeste. Portanto, o principal ator institucional da gestão foi a Secretaria Municipal de Saúde, como já evidenciado ${ }^{3}$. A explicação tem sido a realização de convênios diretamente entre municípios e Ministério da Saúde. "Tem UPA que vem pela rede e tem UPA que o prefeito vai lá no MS e pede: 'eu quero UPA'e a UPA vem” (E15).

Mesmo as UPA planejadas muitas vezes não estão sob gestão estadual por escolha do gestor. "O estado quer assumir o que é do estado, os hospitais" (E15).

Houve relato de transferência de gestão do município para o estado. Uma explicação foi a "falência" dos municípios em assumir a gestão das UPA, havendo a necessidade de devolver essas unidades para gestão estadual.

Evidenciou-se o predomínio de localização da UPA no interior (Tabela 2). Estudo nacional ${ }^{3}$ indica o predomínio de UPA em municípios populosos. Com a atual concentração no interior, pode-se inferir uma mudança nessa concentração de UPA em grandes municípios.

Tabela 2. Número de UPA por estado, localização, porte e esfera de gestão, 2016.

\begin{tabular}{|c|c|c|c|c|c|c|c|c|}
\hline \multirow{2}{*}{ Unidades federativa } & \multirow{2}{*}{ Total de UPA } & \multicolumn{2}{|c|}{ Local } & \multicolumn{3}{|c|}{ Porte de UPA } & \multicolumn{2}{|c|}{ Esfera de gestão } \\
\hline & & Capital & Interior & I & II & III & Municipal & Estadual \\
\hline \multicolumn{9}{|l|}{ Região Norte } \\
\hline Acre & 2 & 2 & 0 & 1 & 0 & 1 & 0 & 2 \\
\hline Amapá & 1 & 1 & 0 & 1 & 0 & 0 & 0 & 1 \\
\hline Amazonas & 1 & 1 & 0 & 0 & 0 & 1 & 0 & 1 \\
\hline Pará & 11 & 1 & 10 & 2 & 5 & 4 & 11 & 0 \\
\hline Rondônia & 2 & 2 & 0 & 0 & 2 & 0 & 2 & 0 \\
\hline Roraima & 0 & 0 & 0 & 0 & 0 & 0 & 0 & 0 \\
\hline Tocantins & 6 & 3 & 3 & 1 & 5 & 0 & 6 & 0 \\
\hline Total & 23 & 10 & 13 & 5 & 12 & 6 & 19 & 4 \\
\hline \multicolumn{9}{|l|}{ Região Nordeste } \\
\hline Alagoas & 8 & 1 & 7 & 3 & 3 & 2 & 1 & 7 \\
\hline Bahia & 29 & 8 & 21 & 14 & 6 & 9 & 26 & 3 \\
\hline Ceará & 26 & 9 & 17 & 10 & 8 & 8 & 3 & 23 \\
\hline Maranhão & 11 & 4 & 7 & 1 & 7 & 3 & 1 & 10 \\
\hline Paraíba & 10 & 2 & 8 & 6 & 3 & 1 & 6 & 4 \\
\hline Pernambuco & 18 & 5 & 13 & 3 & 1 & 14 & 3 & 15 \\
\hline Piauí & 2 & 1 & 1 & 1 & 0 & 1 & 1 & 1 \\
\hline Rio Grande do Norte & 6 & 2 & 4 & 2 & 3 & 1 & 5 & 1 \\
\hline Sergipe & 4 & 0 & 4 & 4 & 0 & 0 & 3 & 1 \\
\hline Total & 114 & 32 & 82 & 44 & 31 & 39 & 49 & 65 \\
\hline \multicolumn{9}{|l|}{ Região Centro-Oeste } \\
\hline Distrito Federal* & 6 & \multicolumn{2}{|c|}{6} & 0 & 0 & 6 & \multicolumn{2}{|c|}{6} \\
\hline Goiás & 14 & 2 & 12 & 4 & 5 & 5 & 14 & 0 \\
\hline Mato Grosso & 4 & 1 & 3 & 2 & 1 & 1 & 4 & 0 \\
\hline Mato Grosso do Sul & 7 & 4 & 3 & 2 & 2 & 3 & 7 & 0 \\
\hline Total & 31 & 13 & 18 & 8 & 8 & 15 & 25 & 6 \\
\hline \multicolumn{9}{|l|}{ Região Sudeste } \\
\hline Espírito Santo & 4 & 0 & 4 & 0 & 3 & 1 & 4 & 0 \\
\hline Minas Gerais & 45 & 6 & 39 & 11 & 9 & 25 & 45 & 0 \\
\hline Rio de Janeiro & 70 & 32 & 38 & 1 & 10 & 59 & 38 & 32 \\
\hline São Paulo & 99 & 2 & 97 & 39 & 41 & 19 & 99 & 0 \\
\hline Total & 218 & 40 & 178 & 51 & 63 & 104 & 186 & 32 \\
\hline \multicolumn{9}{|l|}{ Região Sul } \\
\hline Paraná & 34 & 8 & 26 & 8 & 13 & 13 & 34 & 0 \\
\hline Santa Catarina & 10 & 2 & 8 & 6 & 1 & 3 & 10 & 0 \\
\hline Rio Grande do Sul & 16 & 1 & 15 & 7 & 5 & 4 & 13 & 3 \\
\hline Total & 60 & 11 & 49 & 21 & 19 & 20 & 57 & 3 \\
\hline Total Brasil & 446 & 106 & 340 & 129 & 133 & 184 & 336 & 110 \\
\hline
\end{tabular}

Fonte: Departamento de Atenção Hospitalar e Urgência (DAHU)/Ministério da Saúde e Sala de Apoio a Gestão Estratégica (SAGE) 2016.

UPA: unidades de pronto atendimento

* Distrito Federal: UPA em Brasília classificada como capital e de gestão estadual com finalidade de computar a soma. 
Nas regiões Norte e Centro-Oeste existe mais equilíbrio entre localização na capital e interior. A maioria das UPA é de porte III, mas existe um equilíbrio nacional entre os portes. Na região Norte, há mais UPA de porte II e na Nordeste, mais UPA de porte I (Tabela 2).

A regionalização é outra estratégia fundamental para o interior onde os municípios não cumprem o critério populacional de 50 mil habitantes. O compartilhamento dos recursos tem se mostrado um desafio. "UPA regional não existe porque só um município recebe recursos apesar dos acordos feitos” (E8).

Outro desafio é compatibilizar esse componente com os anteriores à UPA, com ampla aceitação popular, independentemente da efetividade. "Hospitais de pequeno porte nos municípios que não deveriam existir. Talvez virar UPA. Mas não conseguem fechar o hospital no município com 10, 20 mil habitantes" (E22).

O gestor estadual constrói seus planos de urgência e o gestor municipal define de forma autônoma suas prioridades, mas sofre influência política na defesa de interesses locais ${ }^{17,18}$.

Os estados divergiram sobre a importância da UPA para suas redes, e os municípios fizeram sua opção, mas muitas vezes não tiveram recursos para mantê-la.

O Quadro 3 apresenta falas complementares sobre as categorias analisadas.

\section{CONSIDERAÇÕES FINAIS}

Atualmente nos deparamos com um projeto de atenção às urgências que pretende ser integrador; porém, sua implementação ocorreu de forma fragmentada e com frágil articulação entre os seus componentes.

A indução federal normativa e financeira gerou respostas contraditórias, em que alguns estados consideram a UPA como prioritária e outros, não. É necessária a revisão do atual número de UPA em construção, prevenindo o aumento de UPA sem funcionar. As 620 UPA em construção devem ser analisadas nos espaços gestores previstos no SUS, como os grupos condutores, incluindo o ente federal na negociação após planejamento e revisão da expansão.

Quadro 3. Falas complementares de gestores por categoria analítica.

\begin{tabular}{|c|c|c|}
\hline Categoria & Falas ilustrativas & Síntese analítica \\
\hline \multirow{3}{*}{$\begin{array}{l}\text { Implantação e expansão } \\
\text { das unidades de pronto } \\
\text { atendimento (UPA) }\end{array}$} & $\begin{array}{c}\text { "O valor que o ministério da saúde e estado vão passar...esse custeio ainda } \\
\text { é muito alto para o município" (E13) }\end{array}$ & Subfinanciamento federal \\
\hline & $\begin{array}{l}\text { "O estado não está interessado. Acho que o serviço pré-hospitalar que ele tem é o suficiente e } \\
\text { que mais um componente, a UPA, não vai mudar muito" (E9) }\end{array}$ & Rejeição do estado \\
\hline & $\begin{array}{c}\text { "Eu vejo a UPA como modelo evoluído dos pronto-atendimentos que não tinham } \\
\text { resolutividade. Desde espaço físico, qualificação de equipe, equipamento, medicamento, o } \\
\text { modelo de UPA é mais adequado" (E7) }\end{array}$ & Aceitação do estado \\
\hline \multirow{2}{*}{$\begin{array}{l}\text { As condições estruturais } \\
\text { para o atendimento }\end{array}$} & $\begin{array}{l}\text { "Bem estruturada, melhorou as condições de trabalho dos profissionais" (E5) } \\
\text { "Tem UPA que é muito melhor que a urgência do hospital" (E24) }\end{array}$ & Boas condições estruturais \\
\hline & "Falta de pediatra, grave problema" (E13) & $\begin{array}{l}\text { Falta de profissionais } \\
\text { médicos }\end{array}$ \\
\hline \multirow{3}{*}{$\begin{array}{l}\text { A UPA e outros } \\
\text { componentes da } \\
\text { rede de urgência }\end{array}$} & $\begin{array}{l}\text { "No interior, os municípios pequenos tem } 100 \% \text { de cobertura da ESF, mas o médico atende 1, } \\
2 \text { dias na semana" (E6) }\end{array}$ & $\begin{array}{l}\text { Dificuldade na relação com } \\
\text { a atenção básica }\end{array}$ \\
\hline & "O estado queria ter leitos de UTI nas UPA" (E5) & Falta de leitos hospitalares \\
\hline & $\begin{array}{c}\text { "Hospital espelho - hospital de referência metropolitana com HPP ou filantrópicos se integram } \\
\text { para definir farmácia, rotatividade de leitos etc" (E11) }\end{array}$ & $\begin{array}{l}\text { Estratégias para } \\
\text { enfrentamento dificuldades } \\
\text { na gestão hospitalar }\end{array}$ \\
\hline \multirow[t]{2}{*}{$\begin{array}{l}\text { A gestão da UPA } \\
\text { e a interação entre } \\
\text { os entes federados }\end{array}$} & $\begin{array}{l}\text { "UPA regional não faz sentido. Todos os municípios têm um pequeno hospital que tem que } \\
\text { resolver mais que a UPA. O município deixa de investir num serviço próprio para investir em } \\
\text { outro regional que não tem resolutividade maior. Seria diferente se fosse um hospital regional } \\
\text { especializado que resolver mais que o município é capaz" (E9) }\end{array}$ & $\begin{array}{l}\text { Dificuldade de acordo entre } \\
\text { municípios }\end{array}$ \\
\hline & $\begin{array}{c}\text { "Município pediu UPA em 2009, mas desistiu e devolveu o recurso. O estado está resistindo } \\
\text { em assumir porque o parque pré-hospitalar que tem é suficiente" (E6) }\end{array}$ & $\begin{array}{l}\text { Dificuldade de acordo entre } \\
\text { município e estado }\end{array}$ \\
\hline
\end{tabular}


O fortalecimento da gestão estadual foi indicado como desafio para a implantação da rede, além do subfinanciamento e a gestão de pessoas.

Foi benéfico o acesso possibilitado pela UPA, mesmo considerando que sua demanda reflete a incipiência da atenção primária e da rede hospitalar. Esse componente tem o mérito de ter recursos tecnológicos e ser arquitetonicamente diferenciado, mas para ser bem-sucedido e integrado em uma rede potente é necessário investimento na atenção primária e em hospitais.

\section{REFERÊNCIAS}

1. Bittencourt RJ, Hortale VA. Intervenções para solucionar a superlotação nos serviços de emergência hospitalar: uma revisão sistemática. Cad Saude Publica. 2009;25(7):1439-54. https://doi.org/10.1590/S0102-311X2009000700002

2. Cone DC, Irvine KA, Middleton PM. The methodology of the Australian Prehospital Outcomes Study of Longitudinal Epidemiology (APOStLE) Project. Prehosp Emerg Care. 2012;16(4):505-12. https://doi.org/:10.3109/10903127.2012.689929

3. Conselho Nacional de Secretários de Saúde. Rede de Atenção às Urgências e Emergências: avaliação da implantação e do desempenho das Unidades de Pronto Atendimentos (UPAs). Brasília (DF): CONASS; 2015 [citado 31 ago 2017]. (CONASS Documenta, 28). Disponível em: http://www.conass.org.br/biblioteca/pdf/Conass_Documenta_28.pdf

4. De Negri A, organizador. Cartografia do Sistema Hospitalar Brasileiro (2005-2014). São Paulo: LIGRESS/HCor; 2014.

5. Giddens A. A constituição da Sociedade. 3. ed. São Paulo: Martins Fontes; 2009.

6. Konder MT, O’Dwyer G. As Unidades de Pronto-Atendimento na Política Nacional de Atenção às Urgências. Physis. 2015;25(2):525-45. https://doi.org/ 10.1590/S0103-73312015000200011

7. Konder MT, O'Dwyer G. A integração das Unidades de Pronto Atendimento (UPA) com a rede assistencial no município do Rio de Janeiro, Brasil. Interface (Botucatu). 2016;20(59):879-92. https://doi.org/10.1590/1807-57622015.0519

8. Liew Don, Liew Danny, Kennedy MP. Emergency department length of stay independently predicts inpatient length of stay. Med J Aust. 2003;179(10):524-6.

9. Lima LD, Machado CV, O’Dwyer G, Baptista TWF, Andrade CLT, Konder MT. Interdependência federativa na política de saúde: a implementação das Unidades de Pronto Atendimento no estado do Rio de Janeiro, Brasil. Cienc Saude Coletiva. 2015;20(2):595-606. https://doi.org/10.1590/1413-81232015202.02512014

10. Machado CV, Salvador FGF, O’Dwyer G. Serviço de Atendimento Móvel de Urgência no Brasil: uma análise da política nacional. Rev Saude Publica. 2011;45(3):519-28. https://doi.org/10.1590/S0034-89102011005000022

11. Machado CV, Lima LD, O'Dwyer G, Andrade CLT, Baptista TWF, Pitthan RGV. et al. Gestão do trabalho nas Unidades de Pronto Atendimento: estratégias governamentais e perfil dos profissionais de saúde. Cad Saude Publica, 2016;32(2):e00170614. https://doi.org/10.1590/0102-311X00170614

12. Marcolino MS, Brant LCC, Araujo JG, Nascimento BR, Castro LRA, Martins P, et al. Implantação da linha de cuidado do infarto agudo do miocárdio no município de Belo Horizonte Arq Bras Cardiol. 2013;100(4):307-14. https://doi.org/10.5935/abc.20130054

13. Ministério da Saúde (BR). Portaria MS/GM n 1600, de 7 de julho de 2011. Reformula a Política Nacional de Atenção às Urgências e institui a Rede de Atenção às Urgências no Sistema Único de Saúde (SUS). Diario Oficial Uniao. 8 jul 2011; Seção 1.

14. Ministério da Saúde (BR). Portaria $n^{\circ} 2.648$, de 7 de novembro de 2011. Redefine as diretrizes para implantação do Componente Unidade de Pronto-atendimento (UPA 24h) e do conjunto de serviços de urgência 24 (vinte e quatro) horas da Rede de Atenção às Urgências, em conformidade com a Política Nacional de Atenção às Urgências. Diario Oficial Uniao. 8 nov 2011; Seção1:48.

15. O'Dwyer G, Konder MT, Machado CV, Alves CP, Alves RP. The current scenario of emergency care policies in Brazil. BMC Health Serv Res. 2013;13:70. https://doi.org/10.1186/1472-6963-13-7016. 
16. Pires MRGM, Göttems LBD, Cupertino TV, Leite LS, Vale LR, Castro MA, et al. A utilização dos serviços de atenção básica e de urgência no SUS de Belo Horizonte: problema de saúde, procedimentos e escolha dos serviços. Saude Soc. 2013;22(1):211-22. https://doi.org/10.1590/S0104-12902013000100019

17. Santos AM, Giovanella L. Governança regional: estratégias e disputas para gestão em saúde. Rev Saude Publica. 2014;48(4):622-31. https://doi.org/10.1590/S0034-8910.2014048005045

18. Silva EC, Gomes MHA. Impasses no processo de regionalização do SUS: tramas locais. Saude Soc. 2013;22(4):1106-16. https://doi.org/10.1590/S0104-12902013000400013

19. Tanaka OY, Drumond Junior M, Cristo EB, Spedo SM, Pinto NRS. Uso da análise de clusters como ferramenta de apoio à gestão no SUS. Saude Soci. 2015;24(1):34-5. https://doi.org/10.1590/S0104-12902015000100003

20. Ugá MAD, López EM. Os hospitais de pequeno porte e sua inserção no SUS. Cienc Saude Coletiva. 2007;12(4):915-28. https://doi.org/10.1590/S1413-81232007000400013

21. Wang HE, Mann NC, Jacobson KE, Dai M, Mears G, Smyrski K, Yealy DM. National characteristics of emergency medical services responses in the United States. Prehosp Emerg Care. 2013;17(1):8-14. https://doi.org/10.3109/10903127.2012.722178

Financiamento: Programa de Apoio à Pesquisa, Desenvolvimento e Inovação em Saúde Pública da Escola Nacional de Saúde Pública, Fundação Oswaldo Cruz (Inova ENSP 2013) e Fundação de Apoio à Pesquisa do Estado do Rio de Janeiro (FAPERJ - Processo 216672, 2013).

Contribuição dos Autores: Concepção, planejamento, coleta e análise dos dados e elaboração do manuscrito: GO, MTK. Coleta e análise dos dados, elaboração do manuscrito; LR. Análise dos dados e elaboração do manuscrito: MGML, DFA, GFA. Todos os autores aprovaram a versão final do manuscrito e assumem a responsabilidade pública pelo seu conteúdo.

Conflito de Interesses: Os autores declaram não haver conflito de interesses. 